\title{
NUEVAS APROXIMACIONES A LAS ANTÍGONAS IBEROAMERICANAS
}

El título de un texto, escribía hace tiempo Gérard Genette, es el espacio paratextual en el que se instaura un contrato con el lector, cuya finalidad es orientar su horizonte de la expectativa. Ahora bien, aunque el título de esta nota es bastante explícito, para evitar que esas expectativas superen mi capacidad para satisfacerlas y aun a riesgo de ser redundante, creo conveniente señalar que éste se orienta, especialmente, a algunos objetivos cuyos límites quiero precisar: en primer lugar, intento llamar la atención sobre la reiterada omisión, fácilmente constatable, en que incurren los autores que estudian la pervivencia de la tradición clásica grecolatina en las literaturas modernas de Occidente (incluyendo, como es ya tradicional, y aunque sea tema a discutir, la producción dramática), y raramente se ocupan de textos iberoamericanos, fenómeno del cual es una nueva manifestación el estudio, espléndido pese a ello, que George Steiner dedicó a las Antígonas ${ }^{1}$ y cuya riqueza lo convirtió en guía fundamental de estas reflexiones. En segundo lugar, quiero subrayar que se trata de "aproximaciones" 2 , denominación por la que opté para destacar el carácter provisional de estas primeras conclusiones ya que, como advierto en el título, son "nuevas", su novedad sólo supera a las primeras en lo que se refiere, sobre todo, al número de obras que constituyen el corpus estudiado ${ }^{3}$.

${ }^{1}$ Gedisa, Barcelona, 1987.

${ }^{2}$ Este artículo es una continuación de "Aproximaciones al estudio de Antígona Vélez, de Leopoldo Marechal, y de Antígona furiosa, de Griselda Gambaro”, Praesentia, 1 (1996).

${ }^{3}$ Me parece oportuno señalar que se trata de una segunda incursión en el estudio de las relaciones entre la producción narrativa y dramática de Iberoamérica y la tradición grecolatina, tras la que tuve como objetivo analizar la presencia y la funcionalidad del motivo del "Viaje al Averno" en tres novelas de autores de esas latitudes: Adán Buenosayres de Leopoldo Marechal, Pedro Páramo de Juan Rulfo, y Cubagua de Enrique Bernardo Núnez. En ambas incursiones, por fin, apelé a la orientación teórica propuesta por GÉrard GenetTe en sus Palimpsestes (Éds. du Seuil, Paris, 1982) que, como es sabido, reconoce como eje fundamental la noción de transtextualidad. 
El estudio de lo que, en principio, consideré transposiciones iberoamericanas de la Antígona de Sófocles supone varios problemas de distinta importancia, entre los cuales sobresalen dos: uno es la "naturaleza" de lo que Aristóteles denominaba fábula (traducción del término que él en verdad emplea, "mito", es decir historia) y que estaría en el origen de la tragedia sofóclea; sería, pues, su hipotexto probable. Pero, ¿es en verdad un mito, es decir una historia sagrada, lo que encontramos en Sófocles? ¿No es un "tema", o un "motivo"? El otro problema de importancia no menor es el de la determinación del tipo de relación existente entre las Antígonas iberoamericanas y el texto sofócleo (dejando de lado ahora la relación entre ese texto trágico y aquel relato primigenio al que hice referencia antes). No parece posible pensar en una relación directa entre las transposiciones iberoamericanas y el texto de Sófocles. Lo más atinado es, a mi juicio, pensar en una relación mediata sin que, empero, pueda ahora establecerse fácilmente con una precisión siquiera aproximada la serie de mediaciones existente en cada caso.

Dentro del corpus de piezas teatrales del clasicismo grecolatino cuya transformación dio origen a otras aparecidas a lo largo de dos milenios y medio en la cultura occidental, sobresale, sin duda, la tragedia de Sófocles, que exhibe una abundantísima progenie, como demuestra Steiner. A su análisis, que no pretende exhaustividad, añado las obras de Leopoldo Marechal (Antígona Vélez), Jorge Andrade (Pedreira das Almas), Luis Rafael Sánchez (La pasión según Antígona Pérez) y Griselda Gambaro (Antígona furiosa).

Al trabajar en el tema me preguntaba, igual que Steiner, sobre las causas que podrían explicar las numerosas (al parecer interminables) metamorfosis de la Antígona griega. No parece posible dudar de que, especialmente en este siglo, la recurrencia de temas y motivos grecolatinos ha sido constante y ha asumido diversas configuraciones (filosóficas, antropológicas, psicológicas, literarias). Del mismo modo que para el psicoanálisis y la antropología cultural, como cree Steiner, debemos considerarnos los "hijos de Edipo"; la dramaturgia moderna "de la conciencia y de las identificaciones «simbólicas» nos enfrenta con nuestros hermanos", nuestros "semejantes", como Edipo, Narciso, Prometeo, Ulises. En este siglo se ha puesto de manifiesto una búsqueda de "fuentes", una sed de "comienzos", un retorno a lo arcaico, especialmente griego, que explicaría el fenómeno de la recurrencia, en las culturas del norte. En el continente iberoamericano habría que subrayar la preocupación por no limitar esas fuentes o principios grecolatinos y tener en cuenta a los autóctonos (sobre todo en países como México y Perú, que fueron escenario de espléndidos procesos culturales, sin referencia a los cuales muchos textos posteriores serían inexplicables). 
Aunque parezca una salida y no una solución al problema de la prolífica "descendencia" de Antígona —-sobre el cual reflexionaron filósofos y poetas como Hegel, Goethe, Hölderlin, Brecht—, parece apropiado acudir a la explicación que propone Steiner: "creo que solamente a un texto literario le ha sido dado expresar todas las constantes principales de conflicto propias de la condición de hombre”. Dichas constantes son cinco dicotomías o antinomias así integradas: "1) hombre-mujer; 2) senectud-juventud; 3) individuo-sociedad; 4) vivos-muertos; y 5) hombre-dios (o dioses)", enfrentamientos en los que no hay posibilidad de negociación y por ello generan el insoluble conflicto absolutamente trágico que distingue Lesky (y a los cuales podrían agregarse otros, implícitos o aludidos en los anteriores: pasado-presente, por ejemplo; tradición-innovación, etc.). En su Antígona, Sófocles pone en acción cada una de esas oposiciones: "Que yo sepa - agrega Steiner - ningún otro momento en las creaciones sagradas o seculares de la imaginación alcanza esa totalidad" (pp. 179180). De ahí proviene sin duda su riqueza significativa y la, en apariencia, inagotable posibilidad de actualizarla, de "llamar a la vida" lo que Hölderlin consideraba verdades ocultas, latentes, sin que para ello existan condicionamientos ni de tiempo ni de lugar, aunque, como puede comprobarse, hay tiempos y lugares más propicios para el alumbramiento de nuevos vástagos del linaje. Hölderlin veía a Sófocles y se veía "a sí mismo como poetas en tiempo de crisis, de revolución y de dislocación temporal” (pp. 62-63). En esos tiempos de crisis, precisamente (y el siglo xx, el más "clásico" de todos y el más rico en cataclismos históricos), es que el tema (o motivo, o mito...) de Antígona sirve más eficazmente que ningún otro para plasmar en la poesía la narración o el drama, los más hondos y dolorosos avatares de la existencia del hombre. Su arraigo en las más profundas capas de la condición humana, así sea a partir de un mito, o de un motivo, o de una historia, localizados en el tiempo y en el espacio, hace que, como diría Bajtín, la obra de Sófocles sobre ella construida supere toda acotación y entre en el "gran tiempo" de las más trascendentales manifestaciones dramáticas, lo que explicaría su vigencia en numerosas transformaciones ${ }^{4}$.

Uno de los momentos iniciales del análisis es el que corresponde a la determinación de las relaciones que los textos de Marechal, Andrade, Sánchez y Gambaro mantienen con el de Sófocles. En principio, no es aventurado afirmar que se trata de transposiciones serias, esto es, hipertextos de un mismo hipotexto, cuyas respectivas rela-

\footnotetext{
${ }^{4}$ Es casi ocioso aclarar que estas aproximaciones son producto del análisis de los textos, es decir, del componente "literario" del fenómeno teatral y no tiene en cuenta, salvo escasas excepciones, las precisas indicaciones o didascalias que los dramaturgos consignan para la puesta en escena, así como prescinde también de las posibles iluminaciones que la visión de la misma en cada caso podría aportar.
} 
ciones con éste no pueden ser consideradas tan directas como habitualmente se supone. En otras palabras, parece obligado inquirir si no hay mediaciones, si la experiencia personal que tienen los dramaturgos de la obra de Sófocles no es, como en la generalidad de los casos, según dice Steiner, "un producto del palimpsesto de comentarios y juicios que ahora cubren «el original»". Dicho de otro modo, ¿es posible una lectura "inocente" de Antígona? No existe para nosotros "ninguna Ur-Antigone... nunca podremos remontarnos a la première drama" y por eso lo más inteligente es reconocer que "los juicios sobre Antígona desde Aristóteles a Lacan" (pp. 222-223) forman parte de una experiencia más o menos común. De modo que el paso del hipotexto al hipertexto en los casos ya mencionados (en ninguno, me atrevería a afirmar) no es ni puede ser directo, inmediato, lo que exige a continuación enfrentar el problema de la completa determinación de los "palimpsestos", de comentarios y juicios (y otras obras literarias) que se hallarían en el origen de las de Marechal, Andrade, Sánchez, y Gambaro. Como es evidente, estamos ante la sola enunciación de un problema de difícil solución, un problema que incluye, además, la no menos ardua tarea de descubrir (o ir descubriendo) la interpretación que cada dramaturgo hizo del hipotexto, o más precisamente, del palimpsesto, lectura que orientará sin duda el proceso de la transformación.

Para que mi exposición sea lo más clara posible, presento los aspectos más sobresalientes de la trama de cada una de las piezas estudiadas, con especial énfasis en aquellos que las asemejan o diferencian del presunto hipotexto.

Así, abriendo este ciclo, Antígona Vélez (1951) de Leopoldo Marechal, que desde el título, lo mismo que La pasión según Antígona Pérez y Antígona furiosa, remite explícitamente a la historia de la heroína griega. Se desarrolla en una estancia llamada no casualmente "La postrera", en los confines de la pampa y el desierto argentinos, en el último cuarto del siglo xIx, época de la llamada "conquista del desierto" y ámbito propicio para la tragedia, según estima Luis Martínez Cuitiño. Sin que se sepa claramente la razón, Ignacio Vélez (Polinises) ha desertado de la "civilización" y se ha unido a los indios pampas (la "barbarie"). En el enfrentamiento que sobreviene han muerto Ignacio y Martín Vélez (Eteocles), a quien Facundo Galván (Creonte), tío de los jóvenes, de Antígona y Carmen (Ismena), rinde, como en Sófocles, las debidas honras previas a su sepultura que niega al otro, lo que lo lleva al enfrentamiento con Antígona, en el cual ella contará con el apoyo de su prometido, Lisandro Galván (Hemón), hasta la muerte de ambos, "juntos, y como atravesados por la misma lanza”, según apunta uno de los personajes 5 . No hay

${ }^{5}$ Antígona Vélez, Colihué, Buenos Aires, 1981, p. 77. 
en la obra de Marechal referencias al origen incestuoso de los jóvenes; tampoco hay fraticidio ni se alude a destino o hado alguno. En todo caso, "la pampa, el desierto, la llanura, son los que pesan como una fatalidad sobre los personajes", afirma con acierto Luis Martínez Cuitiño, y de la cual son representantes las tres llamativas brujas que integran el tercero de los coros junto a uno de "Hombres" y otro de "Mujeres"6.

Pedreira das almas (1958) del brasileño Jorge Andrade, tiene algunas coincidencias llamativas con la pieza de Marechal: tampoco hay referencia a la cuestión edípica, ni fraticidio ni apelaciones al destino (aunque en Mariana-Antígona hay, ya avanzada la obra, conciencia de la inevitabilidad de lo que sobrevendrá, porque hay "compromisos superiores a nuestros sentimientos"7). Andrade, además, prescinde de toda señal explícita que remita a la Antígona tradicional para exponer en la escena una serie de acontecimientos ubicados históricamente en 1842, en el sur del Brasil, durante convulsiones políticas, sociales y económicas relacionadas con el cambio de una economía minera a otra de carácter agrícola, desde una perspectiva de la visión imperante en la década de 1950 signada por el subdesarrollo y la dependencia, y sobre la cual Andrade practica "una lectura crítico-poética"8. En ese ámbito, Andrade expone la historia de Urbana, una matrona viuda, conservadora, señora de un pueblo minero, y de su hija, Mariana-Antígona, prometida de Gabriel-Hemón, el líder de una movilización popular en busca de nuevas y mejores condiciones de vida, más justas y más libres; ambas mujeres asumen, en diversos momentos, la defensa de la tradición. En el primer acto, Urbana, que de acuerdo con el esquema habitual más simple podría ser un lejano eco de Yocasta clásica (madre de Mariana-Antígona), defiende en verdad el orden establecido frente a Gabriel, Mariana y sus seguidores (aunque, como señala Carlinda Nuñez, también es el amor lo que la anima). En el segundo acto, por el contrario, Mariana-Antígona, a raíz de la muerte de su hermano Martiniano (un equivalente de Polinices) y de Urbana asume abiertamente la defensa de valores tradicionales, en particular el de dar sepultura a su hermano muerto, pero también de valores democráticos frente al despotismo representado por Vasconcelos-Creonte, jefe militar de la represión, defensor de lo que Mariana llama la ley del

${ }^{6}$ Luis Martínez Cuitiño, "La ley de la llanura y el mito de Antígona", Boletín del Instituto de Teatro, Buenos Aires, 1982, núm. 3, pp. 38-39.

${ }^{7}$ Jorge Andrade, Pedreira das almas. O telescopio, Agir, Rio de Janeiro, 1979, p. 97.

8 Carlinda F. P. Nuñez, O universo trágico da "Antígona" de Sófocles e suas relaçoes com "Pedreira das almas" de Jorge Andrade, Dissertaçao de Mestrado, U.F.R.J., Rio de Janeiro, 1986, p. 159. Nuñez afirma que Pedreira das almas propone "una lectura poética de la mudanza del eje económico nacional de Minas Gerais para San Pablo...”, p. 170 (la traducción es mía). 
"poder de las espadas" (p. 98). No obstante, y a diferencia de las restantes piezas, Pedreira das almas no concluye con la muerte de la protagonista (tampoco la de Gabriel-Hemón): ella permanecerá en Pedreira, junto a sus muertos, y Gabriel irá con sus seguidores en busca del nuevo horizonte.

La pasión según Antígona Pérez (1968) del puertorriqueño Luis Rafael Sánchez instala la historia de Antígona en una ficticia pero verosímil "republiqueta" continental tan común en las décadas de 1950 y 1960 llamada Molina, denominación que es también el apellido de Creón, el "generalísimo Creón Molina", tío de Antígona Pérez y dictador. Aurora, madre de la heroína - viuda de otro militar que poniéndole ese nombre había satisfecho "su inclinación a lo heroico"9, según Antígona, y que en mal momento había jugado "la carta equivocada"-, trata de que la joven revele el lugar en el que ha sepultado los cadáveres de Héctor y Mario Tavárez, los clásicos "subversivos" de la época que atentaban contra el orden "occidental y cristiano", según repetían los regímenes dictatoriales de la "Doctrina de la Seguridad Nacional”. Estos jóvenes habían sido asesinados por los secuaces de Creón y condenados a permanecer insepultos para escarmiento general. Como se habrá advertido, no hay relación familiar entre Antígona Pérez y los Tavárez (tampoco hay referencia al origen incestuoso y menos al fraticidio ni al destino): al comienzo de la obra, Antígona, a cargo del "prólogo", recuerda que los militares amigos de su padre le preguntaban “¿dónde están tus hermanos?”, sin que ella atinara a una respuesta "porque no había aprendido que para llamarse Antígona se necesitan hermanos. Ni sabía que Antígona era la muchacha joven que se decide por la muerte". Pero, ya muertos, dirá que los Tavárez "dejaron de ser mis amigos y se hicieron mis hermanos” (pp. 26, 14 y 27). Antígona Pérez es objeto de un acoso pertinaz en el que participan su madre, una típica "señora bien", sumisa y condescendiente con el poder, el dictador, Pilar Varga, su mujer, el jefe de la Iglesia, monseñor Bernardo Escudero, su amiga Irene Domingo y Zúñiga (¿Ismena?), mediante el cual intentan convencerla, cada uno según su competencia, de que revele el sitio de la sepultura. La total soledad en la que se debate Antígona aumenta porque su prometido, Fernando Curet-Hemón, es nombrado por Creón "teniente coronel con cuartel permanente en palacio" y de él dependerá, paradójicamente, que ella reciba o no visitas. La prensa, por su parte, desempeñando una función más o menos semejante a la del coro trágico, pero en versión brechtiana, además de ubicar los hechos en un momento histórico preciso (anunciando por ejemplo acontecimientos como la muerte de Juan XXIII) colabora obsecuentemente, como era común en esos tiempos, con la dictadura. El desenlace, fatal tam-

${ }_{9}^{2}$ La pasión según Antígona Pérez, Cultural, San Juan de Puerto Rico, 1978, p. 15. 
bién aquí como en Antígona Vélez, se produce como conclusión de un planteamiento un tanto extremo y muy "sesentista". Antígona, fusilada, será, como ella proclama, "otro nombre para la idea viva, obsesionante, eterna de la libertad"; pero para los periodistas de los noticieros - el "ajusticiamiento" de "la facinerosa" Antígona Pérez, "una de las más temibles delincuentes de la república" cumpliendo así "finalmente su cita con la ley" - será una de tantas noticias, la mayoría de corte farandulesco y frívolo, como que Jacqueline Kennedy "pasará sus vacaciones en las montañas suizas", o que Pierre Cardin "ha anunciado una nueva línea para beneplácito del público femenino" (pp. 102, 121 y 122).

A diferencia de las tres piezas precedentes, Antígona furiosa (1986) de Griselda Gambaro es una "versión" notablemente económica, por un lado, y la más "fiel" de todas al hipotexto, por otro, ya que se trata de una transposición de tono paródico (o irónico, dos de los tres tipos de transformaciones con función cómica o "no seria" que reconoce Genette) lo que exige un cuidadoso seguimiento del texto primero para modificarlo mínimamente. El número de personajes en escena es notablemente reducido: Antígona, el Corifeo y Antinoo, y la trama se desarrolla retrospectivamente a partir de la muerte de Antígona. Ella se ha ahorcado, pero — de un modo más o menos similar al de la protagonista de Anouilh ${ }^{10}$, sin la intervención de un Prólogo- cobra vida nuevamente para enfrentar el conflicto al cabo del cual habrá de morir. A los personajes que están siempre en escena se suma una "carcasa que representa a Creonte. Cuando el Corifeo se introduce en ella —señalan las didascalias para la puesta-, asume obviamente el trono y el poder"11. Además, así como Antígona encarna a Hemón en ciertos momentos, el Corifeo y Antinoo, aunque Gambaro prescinde formalmente del coro, parecen cumplir a veces también esa función apareciendo, por ejemplo, como voceros de cierto "clamor popular" que "nace siempre de palabras secretas", según declara Antígona.

Por fin, también en coincidencia con la obra de Anouilh, el espacio y el tiempo no experimentan cambios muy pronunciados. Sería, por tanto, de acuerdo con Genette, homodiegética, sin ignorar, por supuesto, otros indicios o señales culturales actualizadores: el

${ }^{10}$ Las coincidencias apuntadas no permiten pensar en un seguimiento particular de Anouilh por Gambaro. Pero, en todo caso, debe destacarse que en el palimpsesto de obras y comentarios en relación con Antígona, la pieza de Jean Anouilh de 1944 ocupa un lugar preponderante, sobre todo en el teatro de la segunda posguerra. Steiner confiesa que, pese a las objeciones que la Antigone de Anouilh suscita, vio "muchísimas representaciones y alguna parte tuve en ellas". Antigone "invadió" todos los ámbitos educativos y teatrales en la posguerra: "su melancólico desencanto - agrega Steiner-, sus antiheroicas chaquetas de cuero cautivaron tanto a los histéricos como a quienes sentían una supervivencia inmerecida" (p. 221).

11 Antígona furiosa, en Teatro, Eds. de la Flor, Buenos Aires, t. 3, p. 196. 
lenguaje, por ejemplo, los trajes que visten Corifeo y Antinoo, el café que beben, pero, sobre todo, una muy reiterada apelación a la "locura" de Antígona por parte de aquellos personajes. Después de insistir en la prohibición de dar sepultura a Polinices, Corifeo dice: "que nadie gire - se atreva - gire gire como loca dando vueltas frente al cadáver insepulto insepulto insepulto", en franca alusión a las Madres de Plaza de Mayo inicialmente calificadas de "locas" por el proceso dictatorial argentino de los años setenta. A ello podrían añadirse otros ejemplos que apuntan casi explícitamente a la infausta situación política y social de la Argentina de esos años, como el que tiene que ver con las desapariciones: "no estaré con los humanos ni con los que murieron —dice Antígona-, no se me contará entre los muertos ni entre los vivos. Desapareceré del mundo en vida”. Tiresias, finalmente, en su condición de sacerdote es, para Antígona, "hábil para ser amigo del poder en su cúspide y separarse cuando declina..." (pp. 210 y 214).

Ahora bien, ¿cuáles son los mecanismos transformadores mediante los cuales se efectúa en las cuatro obras el tránsito del hipotexto al hipertexto? Según Genette, el hipertexto establece con el hipotexto dos tipos de conexión, la relación, por un lado, que puede ser de carácter transformador, propiamente dicho, o imitativo, y, por otro, el régimen (función o funcionamiento del texto segundo respecto del primero) que puede ser lúdico, satírico o serio. Desde esta perspectiva no puede caber duda de que Antígona Vélez, La pasión según Antígona Pérez y Pedreira das almas son hipertextos de régimen serio que resultan de la transformación y que Antígona furiosa es una transposición no seria, afirmación tentativa sobre una caracterización de la cual no estoy muy seguro (es decir habrá que determinar si corresponde a lo que Genette entiende como parodia o como una transformación irónica). Ese tránsito se produce por medio de una serie de operaciones que, en principio pueden distinguirse cuantitativa y cualitativamente: en el primer caso, por ejemplo, mientras es notorio que las piezas de Marechal, Andrade y Sánchez tienen una extensión más o menos similar a la de la tragedia de Sófocles, la de Gambaro es una reducción que entre las diferentes posibilidades mencionadas por Genette podría ser la que denomina concisión, hechos ambos que, pese a su condición puramente cuantitativa (material), no carecen por supuesto de efecto sobre la significación o significaciones. Entre las operaciones de carácter cualitativo deberán tenerse en cuenta procedimientos como la transmodalización ("cualquier clase de modificaciones realizadas en el modo de representación característica del hipotexto. Cambio de modo... o cambio en el modo, pero no cambio de género"12): las obras de Marechal, Andrade, Sánchez y Gam-

12 GenetTe, op. cit., p. 356. 
baro son, como el hipotexto, dramas, aunque no estén escritas en verso (esto dicho con independencia de las resonancias poéticas de Antígona Vélez y Pedreira das almas, en particular). Así, no estamos ante hipertextos que resultan de la transmodalización; en todo caso, podríamos decir que se trata de transprosificaciones.

Tampoco hay cambios notables en cuanto a la perspectiva (no habría entonces transfocalización), ya que la historia de las vicisitudes expuestas en todos estos dramas es, otra vez, la de Antígona, si bien debe recordarse que en Pedreira das almas el papel protagónico desde cuya perspectiva se exponen los hechos es compartido por madre e hija, Urbana y Mariana, en cada uno de los actos que componen la obra.

Por otra parte, también debe recordarse que, como se dijo, mientras Antígona furiosa es homodiegética, es decir, no hay en ella cambios drásticos de espacio y tiempo, las otras tres son heterodiegéticas: el espacio y el tiempo en que la trama se instala y evoluciona ha experimentado, según se comprobó, modificaciones considerables, las cuales reclaman como correlato importantes cambios en el orden pragmático (la muerte de Antígona Vélez y Lisandro-Hemón por un lanzazo; la de Antígona Pérez por arma de fuego), estos cambios, escasos, operan como medios de actualización de la historia en Antígona furiosa (trajes, café, etc.). El cambio de diégesis, además, obedece al propósito de aproximar la historia antigua al presente, lo que Genette denomina proximización, y que si bien priva a la historia y a los personajes de la dimensión de grandeza que parece ser correlativa del pasado remoto, al mismo tiempo los humaniza y los acerca al hombre común (que Antígona lleve apellidos como Vélez o, sobre todo, Pérez, responde a tal propósito). Esta operación, insisto, supone la necesidad de introducir una serie de modificaciones que acabarán transformando la historia de base. Desde este punto de vista, es indudable que Andrade lleva a cabo la transposición más radical en Pedreira das almas, drama en el que, como se vio, deja de lado todo indicio o señal explícitos que orienten la expectativa del espectador (o del lector) hacia el tema clásico. Ya apunté que ello no ocurre sólo en el título sino que también se extiende a los nombres de los personajes. Es casi redundante consignar que en Pedreira das almas se producen otros cambios de orden pragmático, como el de la nacionalidad del personaje, por ejemplo, rasgo que obviamente comparte con Antígona Vélez y La pasión según Antígona Pérez. Tebas es la estancia "La postrera" en Antígona Vélez, el pueblo de Pedreira en la obra de Andrade, cualquier capital iberoamericana en la de Sánchez, pero en todo caso funcionan como equivalentes a Comala, Macondo o Santa María de la novela, subsumiendo el mundo entero (el iberoamericano en particular) en tales microcosmos. 
En esta línea de análisis surge a continuación otro problema: la motivación de la acción. ¿Hay alguna modificación de aquélla, se introduce una donde parecía no haberla, se sustituye la existente por otra que se considera más apropiada? En otras palabras, ¿es posible advertir en los hipertextos lo que Genette denomina transmotivación? Me parece que la respuesta es negativa. Las piezas estudiadas, como es propio de todo drama, exponen un enfrentamiento, una oposición, una agonía, en suma, y es posible constatar sin mayores vacilaciones que en todos los casos el "agente catalizador de todas las contradicciones" (la oposición civilización-barbarie en Antígona Vélez, el problema de las dictaduras y la lucha por la libertad y el cambio en las otras tres ${ }^{13}$ ), es la prohibición de dar sepultura a un muerto, hermano de sangre de la protagonista en Antígona Vélez, Pedreiras das almas y Antígona furiosa y hermano en la solidaridad humana y política en La pasión según Antígona Pérez. En las obras de Marechal y Andrade el énfasis sobre el punto tiene un carácter marcadamente religioso, lo que no ocurre en las de Sánchez y Gambaro. Antígona Vélez replica a su hermana Carmen-Ismena:

la tierra lo esconde todo. Por eso Dios manda enterrar a los muertos, para que la tierra cubra y disimule tanta pena.

CARMEN — ¡Está prohibido enterrar a Ignacio Vélez!

Antígona -Lo sé. Pero yo conozco una ley más vieja (p. 45).

Para Gonzalo, el sacerdote de Pedreira, “¡es una impiedad dejar un cuerpo sin sepultura! ¡Es una ofensa a los muertos!”. Cuando se violan las leyes de Dios, las únicas —exclama Mariana-Antígona, dirigiéndose a Vasconcelos-Creonte - que "el pueblo de Pedreira das almas aceptará”, y se actúa impíamente: “ ¡los muertos saldrán de sus sepulturas y los impíos serán destruidos! (Los soldados se miran atemorizados, dice la didascalia) ique un anatema caiga sobre sus cabezas! - maldice- ique el cuerpo de mi hermano quede expuesto... será un recuerdo viviente de su pecado, de su indignidad!” (p. 70).

En el proceso transformador que describe cada uno de los hipertextos y que hemos apenas bosquejado, sobresale por su decisiva importancia la operación que Genette denomina transvalorización. En rigor, creo que aunque Genette lo explica como el momento culminante del proceso es, a mi juicio, su causa originaria, la que dirige el proceso de transformación. Este momento implica un cambio en el sistema de valores (religiosos, políticos, morales, etc.) que normalmente sustenta toda obra artística y consiste "no necesaria e inmediatamente... [en] la inversión completa de un sistema de valores (sería el caso de una Antígona que tomase el partido de Creonte..)",

13 Cf. Carlinda F. P. Nuñez, op. cit., p. 191. 
sino "toda operación de orden axiológico que puede afectar el valor explícita o implícitamente atribuido a una acción o a un conjunto de acciones"14. Esta metamorfosis obedece, por supuesto, a una determinada interpretación del hipotexto, complejo problema al que ya se aludió y que, como también es evidente, está siempre acotado por las circunstancias históricas en las que vive el interpretante. ¿Qué tipo de transvalorización genera, orienta y corona el desarrollo de la acción en las obras analizadas? Creo que la respuesta requiere volver a Hölderlin y su hipótesis acerca de la correspondencia entre situaciones sociales críticas y Antígona, determinar si, como creo, esa hipótesis es correcta (se trataría, justamente, del otro tipo de relación transtextual reconocido por Genette, la relación del texto con lo extratextual, la "realidad") que las circunstancias históricas y sociales en las que aparecen la obra de Marechal, por un lado, y las de Sánchez, Andrade y Gambaro por otro, son diferentes y podría afirmarse que la hipótesis de Hölderlin (suficientemente confirmada por Steiner) se cumple de modo más manifiesto en Pedreira das almas, La pasión según Antígona Pérez, y Antígona furiosa que en el de Antígona Vélez.

Cuando Marechal retoma el tema a comienzos de la década de 1950, la situación en América Latina no exhibía signos de crisis de la misma profundidad que la de 1960, 1970 y buena parte de 1980, a las cuales remiten respectivamente las piezas de Andrade, Sánchez y Gambaro. En la de Marechal, si bien puede constatarse la presencia de las antinomias mencionadas al comienzo, las oposiciones parecen hallar una síntesis esperanzadora: Antígona Vélez y Lisandro-Hemón mueren juntos a manos de los indios pampas pero su progenie —enfatiza don Facundo Galván-Creonte-, serán "todos los hombres y mujeres que algún día cosecharán en esta pampa el fruto de tanta sangre" (p. 78). "Pedreira das almas propone por su parte una lectura poética del camino del eje económico nacional”, "focalizando" el conflicto que se desarrolla en unas dos semanas en un pueblo minero del sur de Brasil, cuyos habitantes padecerán las repercusiones políticas y sociales que tal acontecimiento económico tiene, por medio de Urbana y sus hijos, Mariana-Antígona y Martiniano-Polinices. La obra de Andrade constituye, en el fondo, un profundo cuestionamiento de las ideas desarrollistas, del progreso de la época; es un intento de revelación de la auténtica cultura brasileña desde una "postura intelectual", que sólo en apariencia se aleja de "su actualidad para criticarla sin predeterminismos ni colores nacionalistas ni nombres folklorizantes" para lo cual "reaproxima el tema de Antígona" a fin de ofrecer una traducción, sin mimetismo, de un aspecto de la profusa identidad brasileña"15.

14 GenetTe, op. cit., p. 42.

15 C. F. P. NuÑEZ, op. cit., p. 169. 
En la tradición de $E l$ señor presidente de Miguel Ángel Asturias y sin la complejidad con que García Márquez, Roa Bastos y Carpentier reconstruyen la tradicional figura del dictador de estas latitudes, tras años de intensa actividad independentista, con estilo brechtiano, Sánchez formula, como escribe Alice de Kuehne, una verdadera alegoría de alcances continentales: "si se evalúa la obra estrictamente como una alegoría política, Antígona encarna el espíritu sobreviviente de las mundanas entrañas de Puerto Rico. Así, como Creón exige que le digan el lugar donde enterraron los cuerpos (de los hermanos Tavárez), ella le replica: «en su tierra, Generalísimo Creón Molina, en la tierra que corresponde a sus cuerpos, en la tierra donde tu ley no es buena»"16.

A lo largo de este análisis de las Antígonas iberoamericanas es posible comprobar que la relación entre estas obras con lo que habitualmente consideramos la realidad histórica es notablemente estrecha. A mi modo de ver, en el caso de la historia iberoamericana de los últimos cuarenta años, la "transtextualidad extratextual", de la que hablaba Genette en sus Palimpsestes, es aquí tan evidente e ilustrativa que cada una de las piezas estudiadas se erige en hito del curso de esa historia, con mucha mayor fuerza, pienso, en el caso de Argentina. Si Antígona Vélez abre el ciclo con una propuesta esperanzada $^{17}$, Antígona furiosa lo cierra (es también una modesta esperanza) con una visión contundente por lo que se refiere a la condena del llamado eufemísticamente Proceso de Reorganización Nacional, responsable de un crudelísimo genocidio, pero escéptica por lo menos con respecto al futuro. Esta actitud crítica es otra elocuente muestra de la "admirable persistencia (con que) el teatro de Gambaro - escribe Magda Castellvi de Moor ${ }^{18}$ - continúa exponiendo y criticando la perversión del poder, cualquiera que éste sea, y la pasividad de quienes quedan sometidos a merced de la autoridad y la violencia".

La furia de Antígona aludiría no tanto a la tradición del furor del teatro latino, sino más bien al concepto que emplea Hölderlin en sus Anmerkungen, salvando algunos matices muy particulares de la sugerente lectura que el poeta alemán hizo de la Antígona de Sófocles. Antígona "furiosa" no parece exactamente el antitheos, porque a quien se opone es al poder secular, al despotismo y no a Dios o a los

16 A. DE Kuehne, "The Antigone theme in Anouilh, Marechal and Luis Rafael Sánchez", apud Gloria F. Waldman, Luis Rafael Sánchez: pasión teatral, Instituto de Cultura Puertorriqueña, San Juan de Puerto Rico, 1988, p. 305.

${ }_{17}$ Marechal habría escrito la obra por especial pedido del primer gobierno de Perón, aparentemente con el propósito de superar la dura dicotomía peronismoantiperonismo que tan negativas consecuencias tendría.

18 "La parodia en el teatro de Gambaro: interdiscursividad y voluntad de estilo", en Teatro y teatristas. Estudios sobre teatro iberoamericano y argentino, ed. O. Pelletieri, Galerna-Universidad, Buenos Aires, 1992, p. 147. 
dioses: "furia, enojo es ahora la palabra clave —escribe Steiner-. Sólo en la 'furia' (Zorn), una palabra que Hölderlin usa en las $A n$ merkungen y agrega dos veces al texto de la tragedia, puede el apasionado retador de Dios abandonar la compostura 'orgánica' de su ser y soltarse de sus ataduras seculares y cívicas"; "una pasión querida, por uno mismo", habría escrito Hölderlin, "una violenta autonomía del impulso - agrega Steiner - es lo que ha empujado a la hija de Edipo a su propia ruina". El furor es lo que hace posible el autorreconocimiento del Antitheos, como "alguien consumido por los violentos y primigenios fuegos de energía vital que lo relacionan con los dioses [y] lo impulsan a buscar a los dioses en mortal combate". Pero, además, no sólo el personaje está furioso, también el dramaturgo, el poeta. En la interpretación de Hölderlin, subraya Steiner, "están implícitos... los hilos autobiográficos. Hölderlin se reconocía como un espíritu a quien habían puesto ‘furioso' la inspiración y la sordera filistea de la sociedad que lo rodeaba”.

Con economía notable Antígona furiosa vuelve a destacar la soledad de la mujer Antígona que, como las heroínas de la Revolución Francesa o las de la Comuna, o la anarco pacifista de Sófocles-Hölderlin-Brecht o como las de las obras que hemos comentado, se enfrentan al poder, a la sociedad, a la divinidad, reclamando justicia en actitud que ningún hombre sería capaz de asumir ${ }^{19}$. Las referencias al inmediato pasado argentino son claras e indudables: ante el ultraje al que la someten Corifeo y Antinoo, Antígona exclama: "no estaré con los humanos ni con los que murieron, no se me contará entre los muertos ni entre los vivos. Desapareceré del mundo, en vida". A su vez, Corifeo le dice, "bondadosamente" según la indicación del autor: "el castigo siempre supone la falta, hija mía, no hay inocentes". Y agrega, con el apoyo de Antinoo: "Y si el castigo te cayó encima, algo hiciste que no debías hacer". Más adelante, el mismo Corifeo ordena: “...¡Enciérrenla! Que sea abandonada en esa tumba. Si ella desea morir allí, que muera. Si desea vivir sepultada bajo ese techo, que viva. Quedaremos puros de su muerte y ella no tendrá contacto con los vivos", a lo cual el bufonesco Antinoo agrega: "¿Qué sabiduría! Está y no está, la matamos y no la matamos". Como la heroína griega, también la de Griselda Gambaro cree haber nacido para "compartir el amor y no el odio", pero en un mundo en el que impera el despotismo "el odio manda" y sólo quedan el silencio y la muerte. Del mismo modo que en Pedreira das almas y en La pasión según Antígona Pérez, pero con una intensidad indudablemente mayor la insolubilidad de los conflictos planteados ha quedado abrumadoramente confirmada en Antígona furiosa que, tal como reza la didascalia, "Se da muerte. Con furia" (p. 217).

${ }^{19}$ Cf. G. SteIner, op. cit., pp. 78-79. 
Cada uno a su manera, según su sensibilidad y su concepción dramatúrgica y de acuerdo con la magnitud y alcance de los conflictos, ha hecho válidas las palabras de Camus: apelando al mito, siguiendo las huellas de Sófocles y tantos otros, han reaccionado contra el mal y contribuido a fortalecer la memoria colectiva, porque, como para la Antígona de Brecht, las calamidades no pueden ser olvidadas. Al reclamo de Ismena “;Olvida el pasado!”- - responde Antígona: "Eres joven y has visto aún poca crueldad. Ese pasado, que tú quieres que olvide, jamás permitirá que sea olvidado" ${ }^{20}$.

ÁNGEL VILANOVA Universidad de Mérida, Venezuela

20 Bertold Brecht, Antígona, en Teatro completo, Nueva Visión, Buenos Aires, 1967 , t. 13 , p. 78 . 